\title{
Teaching Methodologies for New Information Technologies
}

\author{
João Catarino \\ Dep. of Marketing and Logistics \\ College of Business Administration, Polytechnic Institute of Setúbal \\ Setúbal, Portugal \\ joao.catarino@esce.ips.pt
}

\begin{abstract}
New Information Technologies is a course whose curriculum was developed to fill the need of teaching students with more recent and emerging topics in information technologies. The novelty is the approach taken to group in a single course numerous loosely related subjects and a hybrid problem-based learning teaching methodology. Preliminary empirical evidence encourages us to continue pursuing this approach and we believe that our experiences may help other teachers designing their courses' teaching methodology.
\end{abstract}

Keywords-information technologies, hybrid problem-based learning, curriculum development.

\section{INTRODUCTION}

New Information Technologies is a course of the Information Systems Management study program offered by the College of Business Administration, of the Polytechnic Institute of Setúbal, in Portugal. The course's curriculum was developed to provide students with essential competences in emerging information technologies. Traditionally, some of the subjects covered are taught in one or more dedicated courses. For some of those subjects, prior background knowledge of other topics, like advanced mathematics, is required. To overcome these difficulties, we had to develop an innovative teaching strategy and apply it to a variety of subjects.

\section{A. Background Information}

The Information Systems Management study program is structured as a 3 years bachelor's degree with a workload of 180 credits as defined by the European Credit Transfer and Accumulation System (ECTS), which is an academic credit system for higher education widely used throughout European higher education institutions. The program is also accredited by the Portuguese national Agency for Assessment and Accreditation of Higher Education (A3ES) primarily as a management program and has computer science only as a secondary area of studies. It aims to prepare professionals in business administration and computer science fields, providing skills for the conceptualization, design and implementation of information systems to support innovation and organizational activities. According with the latest report, recent graduates from this program have an employment rate of $96.7 \%$ [1] which indicates a strong demand for professionals in this field.

Within the computer science scientific area of studies, students are required to enroll in courses such as programming, databases and web development similar to what is taught in a software engineering bachelor's degrees. However, the amount of credits available, to the computer science area, are just enough to cover these essential subjects and leave no room for more recent and emerging topics, in which this kind of professionals should also have competences. Moreover some of these emerging topics require advanced knowledge of mathematics which have also a rather limited workload in the program. To better understand this situation it is useful to compare, in terms of ECTS workload, the Information Systems Management program with the Software Engineering program that is also offered by Polytechnic Institute of Setúbal. Table I summarizes both programs' contents by scientific area and emphasizes the constraints described before. As one can notice, in the Information Systems Management program, mathematic workload is half of the Software Engineering program and the computer science workload is one third.

TABLE I. ECTS WORKLOAD COMPARISON BETWEEN BACHELOR'S DEGREE PROGRAMS OFFERED BY THE POLYTECHNIC INSTITUTO OF SETÚBAL

\begin{tabular}{|l|c|c|}
\hline \multirow{2}{*}{ Scientific Area } & \multicolumn{2}{|c|}{ ECTS workload } \\
\cline { 2 - 3 } & $\begin{array}{c}\text { Information } \\
\text { Systems } \\
\text { Management }\end{array}$ & $\begin{array}{c}\text { Software } \\
\text { Engineering }\end{array}$ \\
\hline Mathematics & 15 & 31 \\
\hline Computer Science & 41 & 119 \\
\hline $\begin{array}{l}\text { Information Systems } \\
\text { Management }\end{array}$ & 71 & - \\
\hline Management & 28 & \multirow{2}{*}{19} \\
\hline Law & 4.5 & \\
\hline Economics & 5.5 & \\
\hline Accounting & 5 & 11 \\
\hline Marketing & 5 & $\mathbf{1 8 0}$ \\
\hline Other scientific areas & 5 & \\
\hline $\begin{array}{l}\text { Electrical Engineering } \\
\text { \& Communications }\end{array}$ & - & \\
\hline Total & $\mathbf{1 8 0}$ & \\
\hline
\end{tabular}

Our challenge was to develop a single course that could provide competencies in those emerging information technologies (IT), taking in consideration the previously described specificities of the study program.

The aim of this paper is to describe our approach to the problem and to present our preliminary findings. The remainder of the document is organized as follows. Section II describes the course implementation in terms of format, contents and teaching methodologies. Section III provides a practical example of how a specific subject of the course was taught and in Section IV we present our conclusions. 


\section{COURSE IMPLEMENTATION}

\section{A. Objectives and contents}

Our first activity was to state what were the New Information Technologies course's objectives. We decided that instead of focusing only on the technical side of the information technologies, we would also focus on the added value of these technologies to businesses. As such the course's objectives were summarized in the following topics:

- Provide students with knowledge in current and emerging IT, its importance and potential for businesses.

- Provide students understanding about the strategic importance of the internet and its services for businesses.

- $\quad$ Provide students understanding of electronic markets and IT-based transformation.

- Provide students with hands-on IT skills that could enable them to solve complex problems.

- Provide students with self-learning habits on IT.

This last objective was central to our course. Due the fast pace technological environment lifelong self-learning is absolutely required for these professionals. The subjects being currently covered will be surpassed in the coming years by other technologies, which students will need to learn by themselves.

Next, the course contents were decided. The subjects were decided by the course's instructs based on their professional experience and vision of the most relevant emerging information technologies for those students. The following subjects were chosen:
1) Virtualization and cloud computing
2) Machine learning
3) Chatbot technologies
4) Internet of Things (IoT)
5) Blockchain

We used a systems thinking approach, to choose loosely related subjects that could be studied individually but that could make sense from learning path perspective (e.g. one could think of a cloud computing environment for an IoT application using machine learning as an analytic tool for the gathered data).

At this stage, we also decided the frameworks or environments in which the subjects would be taught:

1) Virtualization: Oracle VM VirtualBox and Microsoft Azure cloud services.

2) Cloud computing and Blockchain: Microsoft Azure cloud services.

3) Machine learning: Python programming language, using Anaconda distribution and Jupyter notebooks.

4) Chatbot technologies: Google Dialogflow.

5) Internet of Things (IoT): Raspberry PI and various sensors.

We were conscious that we had a very ambitious curriculum to cover, due to its extent and complexity of some of the topics and that we would require an appropriate teaching methodology to accomplish our objectives.

\section{B. Teaching Methodology}

In our school, both students and instructors are accustomed with traditional instruction pedagogy, based on theoretical led classes detailing the subjects being taught, followed then by practical and/or laboratory classes where practical application of the theory is covered in a progressive manner. In our course, teaching such a variety of subjects during just a 15 -week semester, would not be possible under this top-down format.

Problem-based learning (PBL) is a teaching methodology which opposes to the traditional instruction pedagogy and uses complex real-world problems to promote learning. PBL was initially used in 1950s to address the exponential expansion of medical knowledge [2]. Nowadays it is used in a variety of schools and different disciplines such as nursing, social health care, law, business, machine industry and architecture [3]. Furthermore, a variety of engineering programs across the world have also implemented this pedagogical methodology [4].

PBL focuses on experiential learning organized around the investigation, explanation, and resolution of problems [5]. The benefits of PBL are well documents in the literature. It stimulates students to take responsibility for their own learning, develops problem-solving skills, promotes effective reasoning, promotes self-directed learning and life-long learning behaviors [2], [3], [5].

In PBL learning begins with a realistic problem tackled by students in a class guided by a tutor who does not lecture but helps the students structure their learning. This methodology is summarized by six core characteristics [6]:

1) The learning needs to be student-centred.

2) The learning has to occur in small student groups under the guidance of a tutor.

3) The tutor acts as a facilitator or guide.

4) The learning starts with an authentic problem.

5) The problems encountered are used as a tool to achieve the required knowledge and the problem-solving skills necessary to eventually solve the problem.

6) Self-directed learning for acquisition of new information.

Our approach to the teaching methodology was to use a hybrid PBL approach, i.e. a mix of the traditional method together with PBL. Several reasons contributed to this decision:

1) The difficulty of some of the subjects.

2) Student's lack of background knowledge in several subjects (e.g. mathematics, computer networks, implementing advanced computer algorithms).

3) Students' limited time for self-studying in so many subjects.

4) Minimizing the associated risks of a new teaching methodology. 


\section{Class Format}

To implement our hybrid PBL approach, the classes were implemented with the following format:

1) A theoretical led class: in this class the instructor introduced the topics under study and provided the a minimum background knowledge. In many cases this background knoledge was transmited in empirical terms only (e.g. a gradient).

2) A laboratory class: in this class the students engaged in two different types of activities

a) Implementing a solution to a real life problem: Students were presented to a real life problem and to its solution. The solution was presented in the form of a tutorial with the objective of complementing the background knowledge provided by the theoretical classes. When implementing the tutorial, the students went trough a guided resolution of the given problem, allowing them to gain additional knowledge. The tutorial also encouraged them to adapt and/or change parts of the solution to understand the most relevant aspects and issues associated with it.

b) Solve a real life problem: after completing the tutorial, students were asked to solve a real life problem by themselves. This is where the PBL approach really initiates and the instructor becomes a tutor in promoting student's active learning and knowledge construction. During the resolution of the problem, the instructor may interrupt, to foster class discussion which can help students digging into certain topics. After the solution is found, the instructor validates the solution with the students.

The ratio of theoretical to laboratory classes was the following: for every hour of theoretical classes there were two hours of laboratory classes.

Despite the use of the hybrid approach, we have kept the basic assumptions of the PBL approach, i.e. during problem analysis, students examine the problem, make inferences based on their prior knowledge and identify questions that need to be answered in order to understand or solve the problem [7]. In the literature we can find other examples of successfully implemented hybrid PBL approaches [8]-[10].

\section{HYBRID PLB IMPLEMENTATION FOR MACHINE LEARNING}

In this section, we will provide an example of our hybrid PBL implementation for a teaching machine learning.

Machine learning is a set of methods that can automatically detect patterns in data, and then use the uncovered patterns to predict future data, or to support decision making under uncertainty [11].

For our course, as an introductory topic on the subject, we decided to focus on multi-layer perceptrons (MLPs), also called feedforward neural networks, which are a specific kind of machine learning. These networks are called neural because they are inspired by how the human brain works to solve complex problems. Fig. 1 presents an illustration of an MLP network.
MLPs are the quintessential deep learning models [12] and of extreme importance to machine learning practitioners as they form the basis of many important commercial applications [12]. Moreover, machine learning methods are usually divided in three types according to the learning method: supervised learning, unsupervised learning and reinforced learning. Of these three types, supervised learning is the most widely used in practice [11] and the type MLPs belong to.

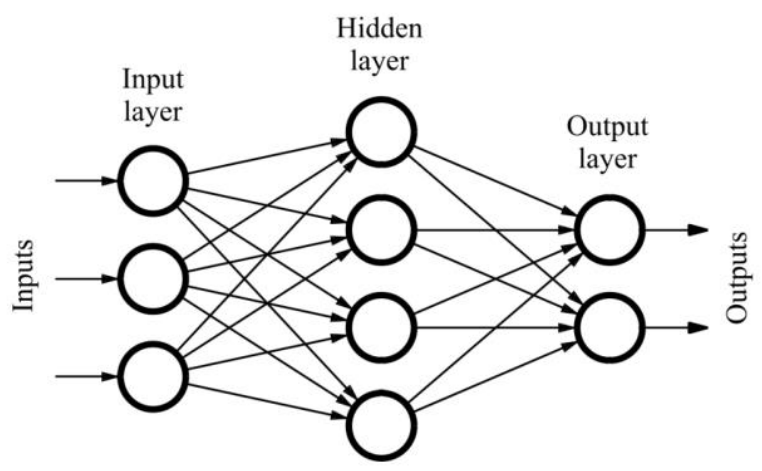

Fig. 1. An MLP network. The network is composed for several units called neurons which are connected with each other. Neurons are organized into layers. These layers are denominated input layer, hidden layer or output layer. A neural network may contain several hidden layers. (source: Nielsen [13])

\section{A. The theoretical classes}

Traditionally, teaching the MLP to students involves going into the mathematical details on how to model and interconnect the neurons of the network, defining a way to measure the models' predictions error, analyzing possible optimization methods to minimize that error and many other related topics that we will not cover into detail in this paper. Readers not familiar with the MLP may refer to reference [12] for an excellent description of the MLP model. All those topics require comprehensive knowledge of linear algebra, differential calculus, optimization and numerical computation which, as explained in the initial section, is beyond our student's knowledge.

Instead of following the traditional teaching approach, we presented those topics on our theoretical class, with minimal mathematical formulation, using extensive empirical examples. Our main goals were to ensure that students understood the possible applications for this machine learning method, how the method worked in practice, the main configurable parameters of the model and their impact on the model's performance. Those were the fundamental aspects that students should acquire to start using MLPs, for solving real world business problems.

\section{B. The laboratory classes}

In the laboratory classes, as a first activity, students implemented a guided solution to a machine learning problem. In this case, the chosen problem was the recognition of handwritten digits. For that, we used a preprocessed dataset called the MNIST [14]. The MNIST dataset consists of 70,000 scans of handwritten digits and associated labels describing which digit 0-9 is contained in 
each image. This machine learning problem is one of the most widely used tests in deep learning teaching and research. Figure 2 and 3 present examples of the scanned digits contained in the dataset.

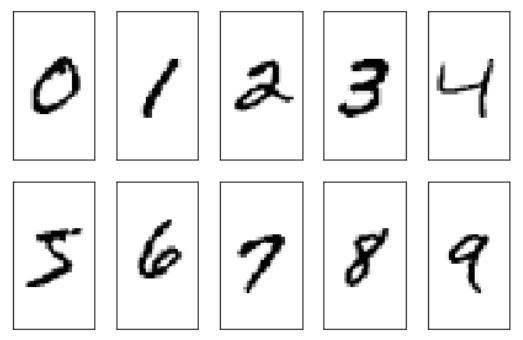

Fig. 2. Randomly selected 0-9 digits contained in the MNIST dataset.

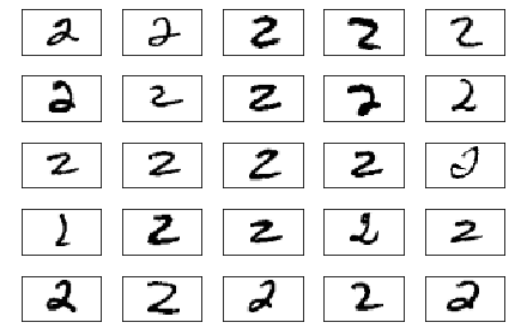

Fig. 3. Some variations of digit 2 contained in the MNIST dataset.

We provided students with a tutorial and didactic implementation of the MLP [15] using the Python programming language. Python is one of the most popular programming languages for data science and has large number of add-on libraries developed specifically for machine learning, that provide not only fast implementations of vector and multidimensional arrays manipulations but also readily implemented high performing machine learning methods.

The tutorial guided the students in implementing the solution to problem. During the tutorial students could get insights on many important practical aspects of the MLP. For illustrative purposes we provide a selection of aspects discussed in our tutorial:

1) How to enconde the handwritten digit into the MLP and its relation with the number of input and output layers.

2) How to evaluate the performance of the implemented $M L P$ and how several parameters (e.g. number of of hidden layers, learning rate, regularization, batches) influence it.

3) Understand the convergence of the MLP, its relation with a cost function and the number of iterations.

4) How to fine tune a MLP to a specific problem. During the tutorial students were encouraged to make variations to the parameter and analyze the impact in the model's performance.

Students could also get graphical insights, like the convergence of the MLP (fig. 4) or visualize some of the digits the MLP struggles to accurately predict (fig. 5).
In the final part of the tutorial, students were guided through the scikit-learn library [16], a popular open source machine learning software library for the Python programming language. Their task was to solve the same problem of recognizing handwritten digits, using this general-purpose library. The library was specially developed to be used by non-specialists, it provides a wide range of machine learning algorithms and is distributed under a BSD license which makes it suitable for both academic and commercial applications. The scikit-learn MLP implementation is higher performing and has a wider variety of parameters to configure, than our didactic implementation. Once again students were encouraged to make variations to the parameter and analyze the impact in the model performance.

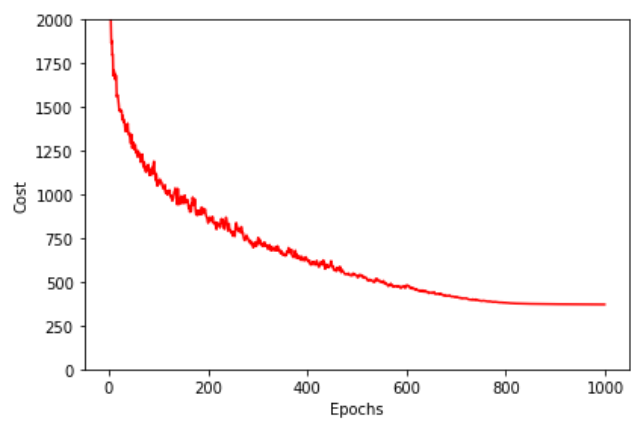

Fig. 4. The MLP cost function for a specific MLP implementation with the MNIST dataset. Students can observe how the cost function converged after roughtly 700 iterations (epochs).

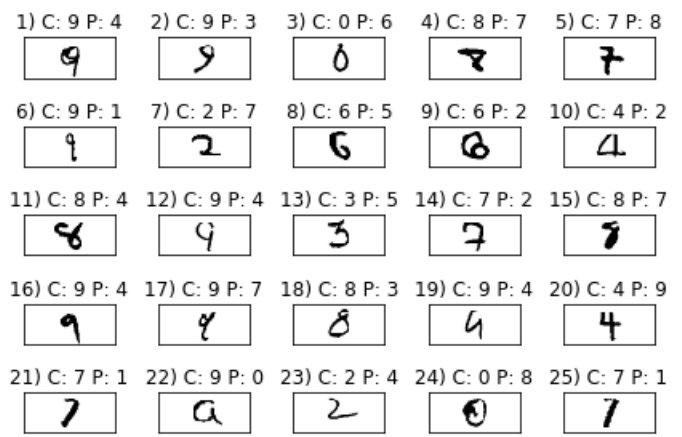

Fig. 5. Visualizing wrongly predicted digits by the MLP. The handwritting can be confusing even for humans. Label ' $\mathrm{C}$ ' on top of each digit represent the correct digit and label ' $\mathrm{P}$ ' on top of each digit represents MLP's prediction of the image.

The second activity of the laboratory classes was to solve a real machine learning problem using the PBL approach. Students were asked to predict human wine taste preferences using two vinho verde datasets [17]. Vinho verde (literally green wine) is a unique wine product from the northwest region of Portugal and gave students an extra stimulus of working with data from a Portuguese product.

The two datasets contain physicochemical objective tests (e.g. PH values) from wines and the respective wine quality score based on sensory data made by wine experts. The experts graded the wine quality between 0 (very bad) and 10 (very excellent) and the final sensory score is given by the 
median of at least 3 evaluations. The first dataset contains 1599 red wine samples and the second dataset contains 4898 white wine samples. Table II presents an overview of the attributes contained on the white wine dataset. The red whine data set contains similar attributes.

TABLE II. ATTRIBUTES CONTAINED IN THE WHITE GREEN WINE DATASET

\begin{tabular}{|l|c|c|c|}
\hline \multicolumn{2}{|c|}{ Attributes } & \multicolumn{3}{c|}{ White wine } \\
\cline { 2 - 4 } & Min & Max & Mean \\
\hline $\begin{array}{l}\text { Fixed acidity (g(tartaric } \\
\text { acid/dm3) }\end{array}$ & 3.8 & 14.2 & 6.9 \\
\hline $\begin{array}{l}\text { Volatile acidity (g(acetic } \\
\text { acid)/dm3) }\end{array}$ & 0.1 & 1.1 & 0.3 \\
\hline Citric acid (g/dm3) & 0 & 1.7 & 0.3 \\
\hline Residual sugar (g/dm3) (g(sodium & 0.6 & 65.8 & 6.4 \\
\hline $\begin{array}{l}\text { Chlorides } \\
\text { chloride)/dm3) }\end{array}$ & 2 & 0.35 & 0.05 \\
\hline Free sulfur dioxide (mg/dm3) & 9 & 440 & 138 \\
\hline Total sulfur dioxide (mg/dm3) & 0.987 & 1.039 & 0.994 \\
\hline Density (g/cm3) (g(potassium & 2.7 & 3.8 & 3.1 \\
\hline pH & 0.2 & 1.1 & 0.5 \\
\hline $\begin{array}{l}\text { Sulphates } \\
\text { sulphate)/dm3) }\end{array}$ & 8 & 14.2 & 10.4 \\
\hline Alcohol (vol.\%) & 3 & 9 & - \\
\hline Quality (sensory score) & & & 35 \\
\hline
\end{tabular}

Students were asked to predict human quality score of the wine datasets using the scikit-learn library. Training the MLP for these wine datasets is substantially harder than for the MNIST. For a start the number of samples is much smaller on the wine datasets and neural networks usually perform much better with large datasets. Secondly, the attributes of the wine datasets have a big variability in their ranges and require preprocessing. Data scientists usually standardize the data to have zero mean and one standard deviation. Decreasing the score intervals for the wine quality can also impact the model performance and may be reasonable for certain business predictions. All these new problems and the guidance to the instructor, served as the basis to acquire new knowledge and problem-solving skills using the PBL approach.

\section{CONCLUSIONS}

We have described the hybrid PBL approach that we developed for the New Information Technologies course. To achieve our objectives, we have devised a hybrid PBL teaching methodology. Despite the use of the hybrid approach, we have kept the basic assumptions of PBL to take advantage of its benefits.

We exemplified our hybrid implementation using as an example the machine learning subject. At the beginning of the classes, students tended to initiate the activities alone. As the activities progressed and the difficulty increased small groups formed spontaneously to discuss possible solutions to the problem. Often, they requested the instructor's guidance on the way to proceed forward. Whenever appropriate, the instructor used those moments to open the discussion to the entire class room. Successful implementation of PBL is critically dependent on the instructor's support to students' active learning [18].

All remaining subjects were taught in a similar manner, except for chatbot technologies where we asked a company specializing in that technology to deliver a seminar. We believe that, whenever possible, bridging the gap between academia and industry makes sense under our teaching model.

Acquiring new knowledge and skills requires a rich learning environment [19]. Only by enabling students to understand and use these new information technologies we can ensure that they will be able to create systems, products, and related services to solve complex problems on organizations. After the first edition of the course, under this format, empirical evidence suggests that students gained enough knowledge to become autonomous in dealing with many aspects of the subjects taught.

This teaching model applies particularly well for courses where time to cover all the topics or insufficient background knowledge are constraints. We believe that our experience may inform and guide other teachers designing their courses' teaching methodology, especially those who intend to teach IT or computer science related topics in programs where computer science is not the primary area of studies.

\section{REFERENCES}

[1] Portuguese Directorate General for Higher Education, "Higher education data and statistics," 2017. [Online]. Available: http://infocursos.mec.pt/dges.asp? code $=3153 \&$ codc $=9630$. [Accessed: 20-Feb-2019].

[2] D. E. Allen, R. S. Donham, and S. A. Bernhardt, "Problem-based learning," New Directions for Teaching and Learning, vol. 2011, no. 128, pp. 21-29, Dec. 2011

[3] R. Pyykkönen and S. Kalliomaa, "PBL applications in the BBA programme in business administration in the School of Business and Services Management at JAMK University of Applied Sciences, Finland," In PBL Across Cultures, K. Mohd-Yusof, M. Arsat, M. T. Borhan, E. de Graaff, A. Kolmos, \& F. A. Phang, Eds., 2013, pp. 1522

[4] J. Peters, Designing inclusion into engineering education: a fresh, practical look at how diversity impacts on engineering and strategies for change. Great Britain: Royal Academy of Engineering, 2018.

[5] C. E. Hmelo-Silver, "Problem-based learning: what and how do students learn?," Educational Psychology Review, vol. 16, no. 3, pp. 235-266, Sep. 2004.

[6] V. V. Shinde, "Designing 'Theory of Machines and Mechanisms' course on project based learning approach," In PBL Across Cultures, K. Mohd-Yusof, M. Arsat, M. T. Borhan, E. de Graaff, A. Kolmos, \& F. A. Phang, Eds., 2013, pp. 163-172

[7] E. Chng, E. H. J. Yew, and H. G. Schmidt, "Effects of tutor-related behaviours on the process of problem-based learning," Adv in Health Sci Educ, vol. 16, no. 4, pp. 491-503, Oct. 2011.

[8] D. E. Allen, B. Duch, S. Groh, G. B. Watson, and H. B. White, "Professional development of university professors: case study from the University of Delaware," in International conference Docencia Universitaria en Tiempos de Cambio [University Teaching in Times of Change] at Pontificia Universidad Católica del Perú, Lima, 2003.

[9] B. J. Duch, S. E. Groh, and D. E. Allen, The power of problem-based learning: a practical "how To" for Teaching Undergraduate Courses in Any Discipline. Stylus Publishing, LLC., 2001.

[10] R. R. Hake, "Interactive-engagement versus traditional methods: a six-thousand-student survey of mechanics test data for introductory physics courses," American Journal of Physics, vol. 66, no. 1, pp. 6474, Jan. 1998.

[11] K. P. Murphy, Machine learning: a probabilistic perspective. Cambridge, Massachusetts London, England: The MIT Press, 2012.

[12] I. Goodfellow, Y. Bengio, and A. Courville, Deep learning. Cambridge, Massachusetts: The MIT Press, 2016.

[13] M. Nielsen, Neural networks and deep learning. Determination Press, 2015 . 
[14] Y. Lecun, L. Bottou, Y. Bengio, and P. Haffner, "Gradient-based learning applied to document recognition," Proceedings of the IEEE, vol. 86, no. 11, pp. 2278-2324, Nov. 1998.

[15] S. Raschka, Python Machine learning. Packt Publishing Ltd, 2015.

[16] F. Pedregosa et al., "Scikit-learn: machine learning in Python," J. Mach. Learn. Res., vol. 12, pp. 2825-2830, Nov. 2011.

[17] P. Cortez, A. Cerdeira, F. Almeida, T. Matos, and J. Reis, "Modeling wine preferences by data mining from physicochemical properties," Decision Support Systems, vol. 47, no. 4, pp. 547-553, Nov. 2009.
[18] S. Bayat and R. A. Tarmizi, "Effects of problem-based learning approach on cognitive variables of university students," Procedia Social and Behavioral Sciences, vol. 46, pp. 3146-3151, Jan. 2012.

[19] B. van der Vlist et al., "Teaching machine learning to design students," in Technologies for E-Learning and Digital Entertainment, vol. 5093, Z. Pan, X. Zhang, A. El Rhalibi, W. Woo, and Y. Li, Eds. Berlin, Heidelberg: Springer Berlin Heidelberg, 2008, pp. 206-217. 